\title{
Two-dimensional nanoultrasonic imaging by using acoustic nanowaves
}

\author{
Kung-Hsuan Lin, Cheng-Ta Yu, Shih-Ze Sun, and Hung-Ping Chen \\ Department of Electrical Engineering, National Taiwan University, Taipei 10617, Taiwan and Graduate \\ Institute of Electro-Optical Engineering, National Taiwan University, Taipei 10617, Taiwan \\ Chang-Chi Pan and Jen-Inn Chyi \\ Optical Sciences Center, National Central University, Chung Li 32054, Taiwan \\ Sheng-Wen Huang and Pai-Chi Li \\ Department of Electrical Engineering, National Taiwan University, Taipei 10617, Taiwan \\ Chi-Kuang Sun ${ }^{\text {a) }}$ \\ Department of Electrical Engineering, National Taiwan University, Taipei 10617, Taiwan and Graduate \\ Institute of Electro-Optical Engineering, National Taiwan University, Taipei 10617, Taiwan
}

(Received 11 March 2006; accepted 1 June 2006; published online 24 July 2006)

\begin{abstract}
Two-dimensional ultrasonic imaging is demonstrated by using acoustic nanowaves. With a $14 \mathrm{~nm}$ acoustic wavelength, both axial and transverse resolutions of a few tens of nanometers are thus achieved. This ultrasonic-based nondestructive technique not only images but also reconstructs the subsurface nanostructures including the depth positions of the buried interfaces. By demonstrating two-dimensional nanoultrasonic scans in depth and transverse (or $z-x$ ) axes, we show that acoustic nanowaves can be a promising tool for future subsurface three-dimensional noninvasive imaging with nanometer resolutions. (C) 2006 American Institute of Physics. [DOI: 10.1063/1.2234723]
\end{abstract}

With rapid advances in nanotechnologies, the demand on noninvasive nanoimaging tools becomes very strong. Nowadays approaches for nanostructure imaging are either restricted to surface measurement or highly destructive. For nondestructive subsurface imaging, ultrasound is a nature chosen alternative. Several notable techniques ${ }^{1-5}$ have combined acoustic technology (in the kilohertz to megahertz regime) and scanning probe microscopy for nanoscale subsurface imaging. Although the scanning probe provides nanometer lateral resolution, the depth and thickness of the buried objects cannot be directly resolved. ${ }^{4}$ To directly map the subsurface structures, a "pulse-and-echo" method has been wildly used in current radio-frequency based ultrasonic systems, ${ }^{6}$ with resolutions limited in the millimeter to micron range. For a nanoscale longitudinal resolution, the wavelength of the launched acoustic pulse should also be in the nanometer scale.

Recently, we found that terahertz longitudinal acoustic nanowaves (or nanoacoustic waves, NAWs) can be optically initiated from periodic piezoelectric nanostructures, ${ }^{7-12}$ named optical piezoelectric transducer (OPT) ${ }^{12}$ Since the OPT can generate and detect terahertz NAW, it makes nanoresolution three-dimensional ultrasonic imaging possible. In this letter, we use InGaN multiple quantum wells (MQWs) as the OPT to demonstrate two-dimensional (2D) ultrasonic nanoscan. The role of the OPT is similar to that of the piezoelectric transducer in a radio-frequency ultrasonic system, while the planar positions of the OPT can be optically controlled externally. As shown in Fig. 1, after NAW was excited by a femtosecond optical pulse and propagated into the object, we used another femtosecond optical pulse ${ }^{12}$ to monitor the time and strength of the echoed acoustic pulses received by the OPT, while the time-variant echo amplitude provides the information about the axial (longitudinal,

\footnotetext{
a) Author to whom correspondence should be addressed; electronic mail:
} sun@cc.ee.ntu.edu.tw $z$-directional) structure of the examined object. On the other hand, for transverse scan (in $x y$ plane), both the examined object and the OPT can be placed on a piezostage. We can thus move the object and the OPT, and perform the corresponding axial scans point by point in the $x y$ plane to obtain and reconstruct 2D or even three-dimensional (3D) images. In our experiments, the temporal OPT sampling rate, which is $15 \mathrm{THz}$ in this study, is much higher than the NAW frequency. The piezostage can also provide nanometer resolution of $x-y$ movement. The resolution of the reconstructed ultrasonic image is consequently determined by the properties of the launched NAW including the acoustic wavelength and pulse width.

To demonstrate 2D nanoultrasonic scan and to investigate the limitations of the longitudinal and lateral resolutions of an ultrasonic image obtained by the NAW, we have designed several test samples. The adopted OPT is a threeperiod $70 \AA / 70 \AA \mathrm{In}_{0.2} \mathrm{Ga}_{0.8} \mathrm{~N} / \mathrm{GaN}$ MQW. The frequency and wavelength of the NAW initiated in this OPT are $0.5 \mathrm{THz}$ and $14 \mathrm{~nm}$. The period of the MQW in the OPT can

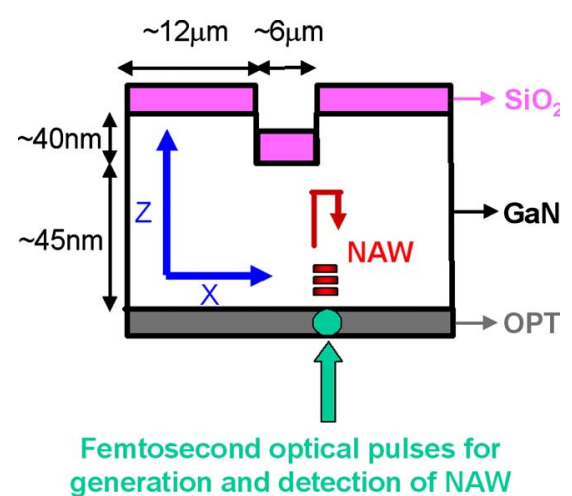

FIG. 1. (Color online) The illustrating diagram for the two-dimensional nanoultrasonic scan by using an optical piezoelectric transducer to generate and detect nanoacoustic waves. Detailed description is presented in the text. 
(a)

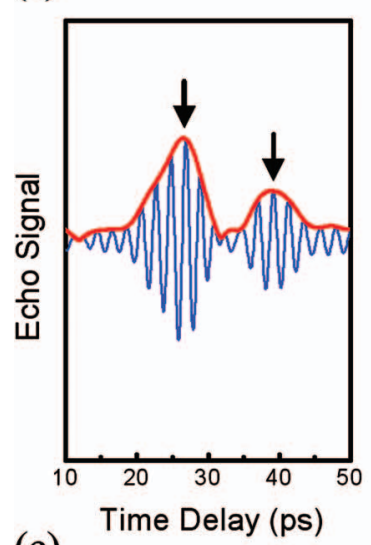

(c)

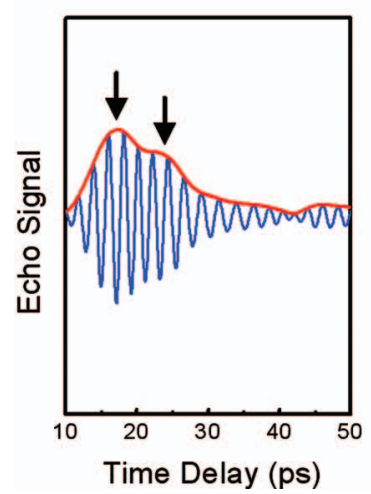

(b)

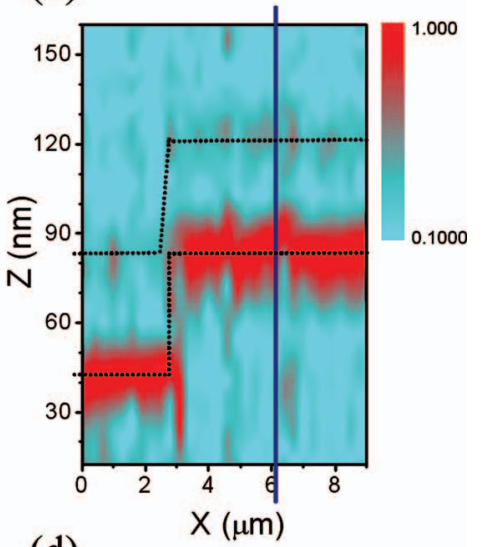

(d)

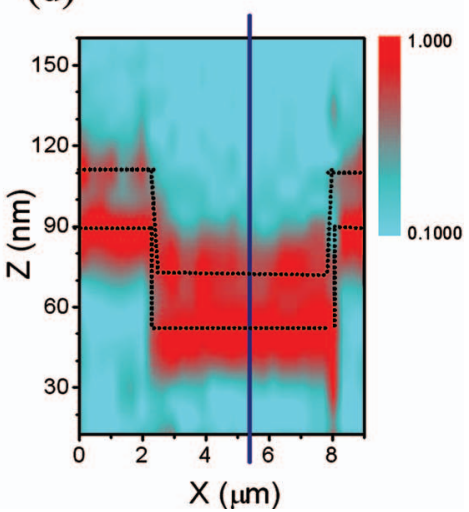

FIG. 2. (Color) The 2D structures of (b) sample A and (d) sample B reconstructed by the 2D nanoultrasonic scans. The dotted lines are depicted for guiding the eyes to locate the positions of the interfaces. The straight lines in (b) and (d) indicate the $x$ positions of the corresponding 1D nanoultrasonic scans shown in (a) and (c). The arrows mark the peak positions of the echoed wave packets due to interface reflections.

be further shortened ${ }^{12}$ for generating higher frequency and shorter pulse width NAW. As shown in Fig. 1, on the top of the OPT, the examined objects were patterned $\mathrm{SiO}_{2}$ and $\mathrm{GaN}$ nanostructures. After the NAWs were initiated in the OPT, they propagated in counterdirections. The NAW propagating toward the examined objects was used for imaging. Since the OPT was grown on a 3.4- $\mu \mathrm{m}$-thick GaN layer on top of a $500-\mu \mathrm{m}$-thick sapphire substrate, the NAW propagating toward the substrate will not interfere with our observed echo signals from the objects in the observed time window.

The thickness of the top GaN layer is in the range between 80 and $90 \mathrm{~nm}$ estimated by the growth rate. The top surface of the GaN layer was etched with a stripe-patterned mask. The pattern had a period of $\sim 18 \mu \mathrm{m}$ including an $\sim 6$ - $\mu \mathrm{m}$-wide etching region. The etching depth was estimated to be $30-50 \mathrm{~nm}$. The patterned GaN layer was covered by a cap $\mathrm{SiO}_{2}$ layer. We prepared three test samples with different thicknesses of the covered $\mathrm{SiO}_{2}$ layers, $\sim 40$, $\sim 20$, and $0 \mathrm{~nm}$ for samples $\mathrm{A}, \mathrm{B}$, and $\mathrm{C}$, respectively.

The experimental setup was similar to a typical pumpprobe system. ${ }^{13}$ The optical pulse width was $\sim 250$ fs with a central wavelength of $400 \mathrm{~nm}$. After optical pump and probe beams were combined by a polarization beam splitter, they were collinearly focused into the OPT with a high numerical aperture (NA) objective. Since the central frequency of the NAW generated in the OPT was $0.5 \mathrm{THz}$, all results of onedimensional (1D) axial scans can be processed by a $0.5 \mathrm{THz}$ bandpass filter. ${ }^{12}$ Figure 2 (a) shows a $1 \mathrm{D}$ scan result in Downloaded 16 Feb 2009 to 140.112 .113 .225 . Redistribution subje

sample A. Two wave packets centered at 26.6 and 38.9 ps delays can be clearly observed. They are due to the reflections of NAW at the $\mathrm{GaN} / \mathrm{SiO}_{2}$ and $\mathrm{SiO}_{2}$ /air interfaces. The amplitude of the first wave packet is higher because the strain reflectivity of $\mathrm{GaN} / \mathrm{SiO}_{2}$ is 0.58 . For typical ultrasounds, the 1D scan results are plotted as a function of time delay to indicate the rough (or relative) structure of the object. If the sound velocity of each layer can be predetermined, the exact structure including the depth can then be reconstructed. Here, the longitudinal sound velocities of GaN and $\mathrm{SiO}_{2}$ are 8 (Ref. 14) and $5.9 \mathrm{~nm} / \mathrm{ps},{ }^{15}$ respectively. From the 1D scan, we can thus obtain the thicknesses of GaN and $\mathrm{SiO}_{2}$ layers to be 86 and $36 \mathrm{~nm}$, respectively.

To obtain the 2D nanoimage of the examined object, we performed 1D scans along the $x$ axis with a step size of $300 \mathrm{~nm}$. Each trace of the 1D scan was normalized to the signal peak in the observed window. Following the thickness calculations, the 2D nanostructure can then be directly reconstructed. The position zero along the $z$ axis was set to the interface of the OPT and the object. In Fig. 2(b), the $\mathrm{GaN} / \mathrm{SiO}_{2}$ and $\mathrm{SiO}_{2}$ /air interfaces at different depths have been resolved. Since the interval of two interfaces was only $\sim 40 \mathrm{~nm}$, this image indicates the high axial resolution when imaging with a NAW. It should be noted that the apparent "width" of the interfaces in Fig. 2(b) is due to the finite pulse width of the echoed NAW. The exact position of the interface can be determined by each $1 \mathrm{D}$ ultrasonic scan as shown in Fig. 2(a) with an axial accuracy of $\sim 1 \mathrm{~nm} .^{12}$

To examine the axial resolving power for two adjacent interfaces, we performed 2D ultrasonic scan on sample B with a reduced interval between two interfaces. The $2 \mathrm{D}$ reconstructed image is shown in Fig. 2(d) and we can still distinguish these two interfaces. Figure 2(c) shows one corresponding $1 \mathrm{D}$ scan at $x=5.4 \mu \mathrm{m}$ and the first and second peaks of the echoed wave packets were located at 17.4 and 22.8 ps. The interval between the $\mathrm{GaN} / \mathrm{SiO}_{2}$ and $\mathrm{SiO}_{2} /$ air interfaces can thus be determined to be $\sim 16 \mathrm{~nm}$, implying that the system capability to resolve two adjacent axial interfaces is better than $16 \mathrm{~nm}$. It is possible to further improve the axial resolving power by using shorter acoustic pulse width or if the interference of the echoed NAWs from two interfaces is to be analyzed. $^{12}$

For the 2D nanoultrasonic scans shown in Fig. 2, we used a NA 0.85 objective. It may be straightforward that the optical spot size of $\sim 350 \mathrm{~nm}$ is the transverse resolution of the $2 \mathrm{D}$ image, since the acoustic spot size is determined by the optical spot size in the OPT. However, following the diffraction limit equation, a lateral acoustic resolution of half the acoustic wavelength ( $7 \mathrm{~nm}$ in this study) should be ideally achievable. ${ }^{16}$ The transverse resolution is not limited by the acoustic spot size, but should be raised by scanning the xy plane with even smaller steps and by analyzing each 1D ultrasonic scan in the time domain.

To explore the transverse resolution of the $2 \mathrm{D}$ nanoultrasonic image, we used a NA 1.35 objective to perform 2D scans on sample $\mathrm{C}$ and compared with a result measured by an atomic force microscope (AFM). The transverse step size was $50 \mathrm{~nm}$, which was much shorter than the generated acoustic spot size of $\sim 200 \mathrm{~nm}$. Figure 3(a) shows the original acoustically reconstructed 2D nanostructure, while different thicknesses of the GaN cap layer are clearly resolved. To investigate the potential lateral resolution of the system taking advantage of the patterned sharp edge, we acquired the to AlP license or copyright; see http://apl.aip.org/apl/copyright.jsp 
(a)

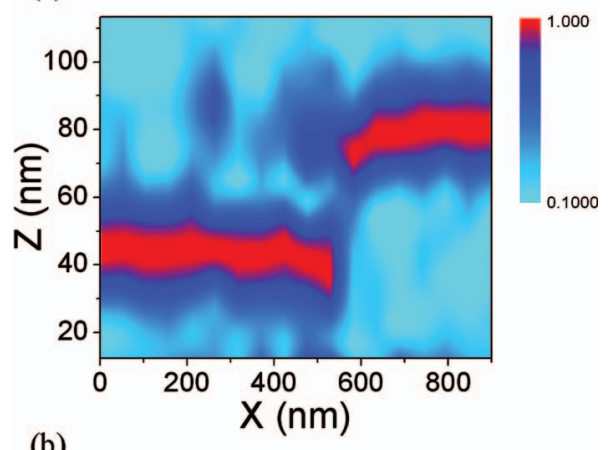

(b)

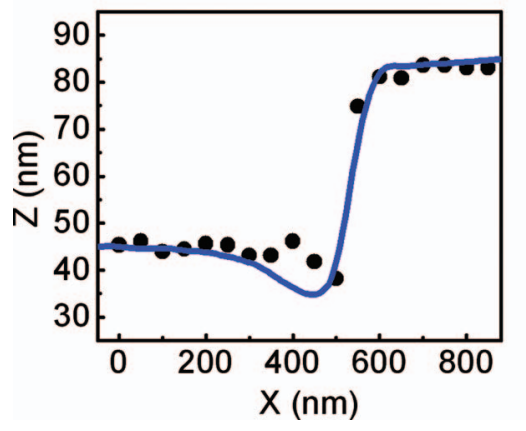

FIG. 3. (Color) (a) The reconstructed 2D structure of sample C measured by the nanoultrasonic scan. (b) By acquiring the peak positions of the echoed wave packets, the imaged 2D structure has been depicted in dots. The measurement result of atomic force microscope is also shown as a solid line for comparison.

peak position of the echo envelope in each 1D scan as shown in Fig. 3(b), which was similar to a deconvoluted 2D image. A high lateral resolution can be found, under a combined analysis with the axial scans. For comparison, a commercial AFM with a $<10 \mathrm{~nm}$ transverse resolution was also utilized to measure the examined surface structure as plotted in solid line in Fig. 3(b). We found excellent agreement between these two measurements. However, the subsurface information such as thickness cannot be obtained by the AFM. It is also interesting that the edge obtained by the nanoultrasonic scan was sharper than that measured by the AFM, due to the finite size of the AFM tip. ${ }^{17}$ Our result clearly provides the experimental evidence that $<100 \mathrm{~nm}$ lateral resolution can be achieved by using a NAW. If we use a step function convolving an effective Gaussian spot to fit the ultrasonic data shown in Fig. 3(b), the transverse resolution, defined by the radius of the effective Gaussian spot, can be found to be better than $50 \mathrm{~nm}$.

Since the wavelength of the NAW is on the nanometer scale, both axial and transverse resolutions of an ultrasonic image can be achieved to be a few tens of nanometers. For such high frequency acoustic nanowaves, the attenuation constant could be on the micron scale at room temperature, ${ }^{18,19}$ thus with a penetration capability of several microns. An ultrasonic image with millimeter-observation depth could be obtained if the temperature is decreased to tens of Kelvin. ${ }^{20,21}$ Through our study, we have clearly demonstrated that NAW is capable to nondestructively obtain a 2D nanoultrasonic image in the $z-x$ axes. To obtain a 3D nanoimage, one can perform additional scans along the $y$ axis. Combing with sub-millimeter wave electronics and nanoscaled electrodes, it is also possible to develop highspeed electronic-based piezoelectric nanotransducers, with an image resolution dependent on the electrode spacing and the frequency and pulse width of the generated NAWs. By simply adopting the fast scanning technology in current optical coherent tomography, an optically based high frame-rate system is also possible. Our study shows that nanoultrasonic imaging could be the promising tool for future noninvasive subsurface 3D imaging with nanometer resolutions.

The authors thank Professor Chih-Kung Lee and Ding Zheng Lin for AFM measurement. This work was sponsored by the National Science Council of Taiwan under Grant No. 94-2120-M-002-004.

${ }^{1}$ K. Yamanaka, H. Ogiso, and O. Kolosov, Appl. Phys. Lett. 64, 178 (1994).

${ }^{2}$ R. E. Geer, O. V. Kolosov, G. A. D. Briggs, and G. S. Shekhawat, J. Appl. Phys. 91, 4549 (2002).

${ }^{3}$ L. Muthuswami and R. E. Geer, Appl. Phys. Lett. 84, 5082 (2004).

${ }^{4}$ A. F. Sarioglu, A. Atalar, and F. L. Degertekin, Appl. Phys. Lett. 84, 5368 (2004).

${ }^{5}$ G. S. Shekhawat and V. P. Dravid, Science 310, 89 (2005).

${ }^{6}$ A. Briggs, Advances in Acoustic Microscopy (Plenum, New York, 1995).

${ }^{7}$ C.-K. Sun, J.-C. Liang, and X.-Y. Yu, Phys. Rev. Lett. 84, 179 (2000).

${ }^{8}$ G.-W. Chern, K.-H. Lin, and C.-K. Sun, J. Appl. Phys. 95, 1114 (2004).

${ }^{9}$ G.-W. Chern, C.-K. Sun, G. D. Sanders, and C. J. Stanton, Top. Appl. Phys. 92, 339 (2004).

${ }^{10}$ C.-L. Hsieh, K.-H. Lin, S.-B. Wu, C.-C. Pan, J.-I. Chyi, and C.-K. Sun, Appl. Phys. Lett. 85, 4735 (2004).

${ }^{11}$ K.-H. Lin, G.-W. Chern, Y.-K. Huang, and C.-K. Sun, Phys. Rev. B 70, 073307 (2004).

${ }^{12}$ K.-H. Lin, G.-W. Chern, C.-T. Yu, T.-M. Liu, C.-C. Pan, G.-T. Chen, J.-I. Chyi, S.-W. Huang, P.-C. Li, and C.-K. Sun, IEEE Trans. Ultrason. Ferroelectr. Freq. Control 52, 1404 (2005).

${ }^{13}$ K.-H. Lin, G.-W. Chern, S.-W. Chu, C.-K. Sun, H. L. Xing, Y. Smorchkova, S. Keller, U. Mishra, and S. P. DenBaars, Appl. Phys. Lett. 81, 3975 (2002).

${ }^{14}$ C. Deger, E. Born, H. Angerer, O. Ambacher, M. Stutzmann, J. Hornsteiner, E. Riha, and G. Fischerauer, Appl. Phys. Lett. 72, 2400 (1998).

${ }^{15}$ T. C. Zhu, H. J. Maris, and J. Tauc, Phys. Rev. B 44, 4281 (1991).

${ }^{16}$ B. C. Daly, N. C. R. Holme, T. Buma, C. Branciard, T. B. Norris, D. M. Tennant, J. A. Taylor, J. E. Bower, and S. Pau, Appl. Phys. Lett. 84, 5180 (2004).

${ }^{17}$ F. J. Giessibl, Rev. Mod. Phys. 75, 949 (2003).

${ }^{18}$ C.-T. Yu, K.-H. Lin, C.-L. Hsieh, C.-C. Pan, J.-I. Chyi, and C.-K. Sun, Appl. Phys. Lett. 87, 093114 (2005).

${ }^{19}$ S. Wu, P. Geiser, J. Jun, J. Karpinski, J. R. Park, and R. Sobolewski, Appl. Phys. Lett. 88, 041917 (2006).

${ }^{20}$ H. Y. Hao and H. J. Maris, Phys. Rev. Lett. 84, 5556 (2000).

${ }^{21}$ B. C. Daly, T. B. Norris, J. Chen, and J. B. Khurgin, Phys. Rev. B 70, 214307 (2004). 